\title{
Smart Polymeric Nanoparticles: Combining Targeted Delivery, Imaging and Therapy into One Nanomedicine Platform \\ Yu Wang*
}

Department of Chemistry, University of Louisiana at Lafayette, LA, USA

\section{Mini Review}

Nanomedicine is the application of nanotechnology in medical care, which refers to highly specific, molecular level medical diagnoses and/ or treatment of disease [1]. Nanomaterials are useful for biomedical applications since the size of nanomaterials, usually $1-1000 \mathrm{~nm}$, is comparable to that of most biological microstructures. The integration of nanomaterials with biomedicine has led to the development of drug delivery systems, diagnostic devices, imaging and therapy applications. Various types of nanomedicine compounds have been used in clinical care, including lipid based nanocarriers, polymer-based nanocarriers, inorganic nanoparticles, and viral vectors [2]. Among those, polymeric nanoparticles are very attractive not only due to their stability, low cost, and predictable characterisation but, more importantly, also due to the structural versatility, and easy incorporation of multiple functions. However, the effect of nanoparticles in human systems is still not well known and there could be concerns about nanoparticle toxicity. Thus, study of the safety of using polymeric nanoparticles in biomedicine is necessary and is an active research topic recently.

Polymeric nanoparticles can incorporate with functionalities easily via organic reactions. Not all coupling reactions can be used in biomedical applications. Desired coupling reactions are those do not interfere native biochemical processes and highly selective towards one functional group in the presence of others. In addition, rapid, high-yield chemistry with simplified purification is required in nanomedicine design. Several types of chemical reactions including click chemistry, thiol-ene or thiol-yne chemistry, and regioselective polyester-drug conjugation chemistry meet all of the requirements and provide versatile tool for polymeric nanomedicine development [3].

The ease of size and shape control is another significant advantage of polymeric nanoparticles. Dendrimers and star polymers are spherical structured, while polymer brushes are closer to cylindrical structures. Those different structures can be obtained via different synthetic methods. Another route for polymeric nanoparticle formation is self-assemble of amphiphilic block copolymers in selected solvents. Various architectures can be formed by adjusting the self-assemble conditions. Size and shape of nanoparticles are critically important for nanomedicine applications [4]. For example, cylindrically shaped filomicelles (diameter 22-60 $\mathrm{nm}$, length 8-18 mm) have shown much longer persistent blood circulation, up to one week after intravenous injection, than their spherical counterparts. Compared to spherical particles, disc-shaped nanoparticles have shown higher in vivo targeting specificity to endothelial cells expressing intercellular adhesion molecule receptors in mice.

Polymeric nanoparticles can incorporate with stimuli sensitive properties via introducing certain functional groups that enable controlled release of drugs under internal or external stimuli. By depositing the active agent only in the targeted region and with proper dose that do not exceed the need, the overall drug consumption and side effects could be reduced significantly. Types of stimuli include $\mathrm{pH}$, photo, thermal, magnetic field, redox potential, and concentrations of enzyme, glucose, etc. An example of internal stimuli responsive system is the incorporation of $\mathrm{pH}$ sensitive groups including cisaconityl amide linkages, hydrazone, oxime, acetal/ketal, trityl, $\mathrm{N}$-ethoxybenzylimidazoles and imino groups, etc. The $\mathrm{pH}$ in tumor tissues and inflammatory tissues is mildly acidic which may trigger drug release from $\mathrm{pH}$ sensitive delivery vehicles once they arrive at the targeted disease sites. One example of external stimuli responsive system is polymeric nanocapsules with magnetic nanoparticles imbedded in. External magnetic field may guide the nanoparticles to concentrate at certain locations. Magnetic iron oxide nanoparticles are the only magnetic nanomaterials approved for clinical use by the US Food and Drug Administration (FDA). Thus, the studies of magnetic nanoparticles for clinical applications mainly focused on those magnetic iron oxide nanoparticles. Iron oxide nanoparticles have good chemical stability, magnetic responsiveness, and biocompatibility, while the preparation method is relatively simple. Beyond the targeted delivery applications, the strong magnetic property of iron oxide makes it well suited for use as an MRI contrast agent. It was shown that even unmodified iron oxide nanoparticles allowed for $90.5 \%$ detection of lymph node metastasis in patients with prostate cancer compared to 35.4\% detection using conventional MRI [5].

Polymeric nanoparticles incorporated with dyes or quantum dots can be used as imaging agents. To understand the distribution and metabolization of drugs, it is important to track their movement. While it is difficult to track a small group of cells throughout the body, one can dye the cells and illustrate the movement of those cells thus can evaluate the distribution and effects of drugs. The small size of nanoparticles endows them with the possibility to incorporate into cells and that can be very useful in imaging. Tailoring the size and structure of the nanoparticles could also help to improve the performance of imaging agents. For example, bright fluorescent was achieved by accommodate thousands of fluorescent dye molecules on a single antibody probe while avoiding the negative effects of self-quenching [6].

Polymeric nanoparticles can be used to encapsulate not only small molecular drugs but also genes. For normally insoluble small molecular drugs, the polymeric carriers can protect them from degradation until

*Corresponding author: Yu Wang, Assistant Professor, Department of Chemistry, University of Louisiana, Lafayette, LA, USA, Tel: 3374826734; E-mail: yuwang@louisiana.edu

Received October 10, 2017; Accepted October 24, 2017; Published October 31, 2017

Citation: Wang Y (2017) Smart Polymeric Nanoparticles: Combining Targeted Delivery, Imaging and Therapy into One Nanomedicine Platform. J Bioanal Biomed 9: 269-271. doi:10.4172/1948-593X.1000191

Copyright: @ 2017 Wang Y. This is an open-access article distributed under the terms of the Creative Commons Attribution License, which permits unrestricted use, distribution, and reproduction in any medium, provided the original author and source are credited. 

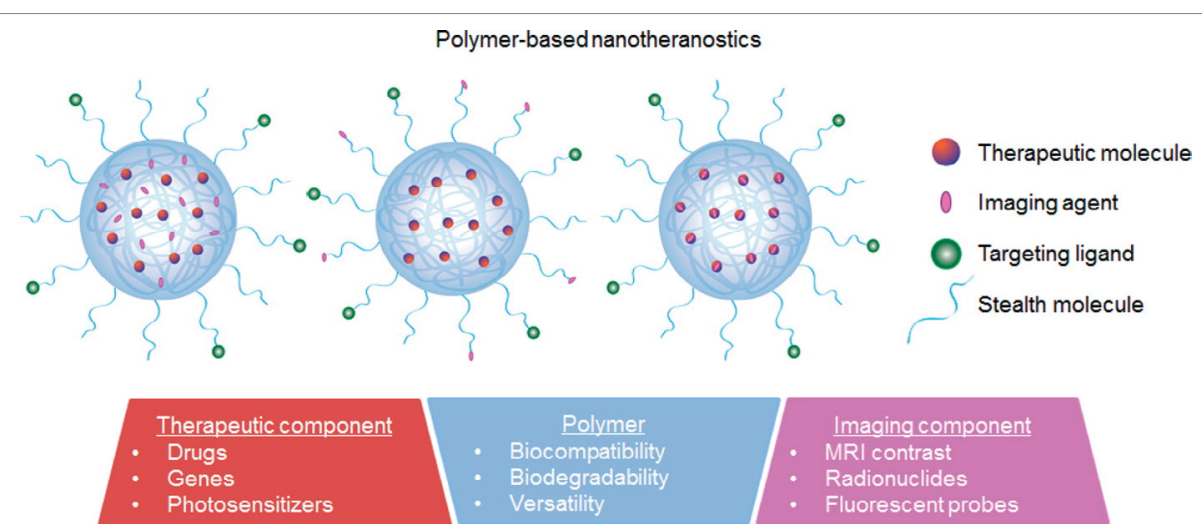

Figure 1: Illustration of a polymeric nanomedicine platform [8]. (Copyright 2014 the American Chemical Society.)

they have reached their target location. For gene delivery, specific genetic materials are transferred to target cells. Through the administration of defined genetic materials (i.e. DNA or siRNA), defective gene can be replaced or regulated, thus a particular disease state could be prevented or altered. Genetic therapy provides promising solutions for next generation therapeutics. Polymeric nanoparticles also find applications in novel therapy development. Recently, it was found that structurally nanoengineered antimicrobial peptide polymers are highly effective in combating multidrug-resistant Gram-negative bacteria [7].

Advanced nanomedicine systems require the combination of targeted delivery, imaging and therapeuticsin a singlenanocarrier, Figure 1. Polymers are especially attractive in multifunctional nanomedicine systems because of the ease of incorporating different functional groups via coupling reactions [8]. Ideally, the Nano system should contain a polymeric carrier component, a therapeutic component, and an imaging component. Targeting ligands for specific delivery may be incorporated in some of those systems, as well. These functional components in the nanomedicine system can be arranged in different ways according to the nano platform as shown in Figure 1. Some therapeutic component may possess an inherent fluorescence, such as doxorubicin [9]. Thus additional imaging component is not necessary. Since the treatment and imaging agents are incorporated in the same nanoparticle, the effect of the therapy can be monitored in real-time. The delivery could be targeted to the capillary bed of the active sites, to the specific type of cell or an intracellular region, or to specific organ/tissues. Mechanisms of targeted delivery include passive targeting and active targeting. In passive targeting, the nanoparticles are cloaked with some sort of coating, e.g., by adding polyethylene glycol (PEG). The body's natural response to physicochemical characteristics of the nanomedicine leads to prolonged circulation time. Active targeting involves several different strategies. Stimuli response to physical or chemical environment, e.g. magnetic field, $\mathrm{pH}$, could be used for targeted delivery as discussed earlier. In one example, a triblock polymer: poly (ethylene oxide)co-poly (Cys-AuNP@FA)-co-poly (3-methoxypropylacrylamide was synthesized as the carrier. Functional components were incorporated into this polymeric nano carrier including magnetic nanoparticles for targeted delivery and as MRI contrast agent for stimuli-responsive T1imaging, anisotropic Au nanoparticle for photothermal therapy. In this study, the authors employed Au nanoparticles with four different shapes. The results showed nanoflower shaped Au nanoparticles is superior to spherical ones in this specific application [10]. In another example, CdTe quantum dots and anticancer drug temozolomide were combined in to a $\mathrm{pH}$ and temperature dual-responsive nano gel composed of hydroxypropylcellulose-poly (acrylic acid) [11].

\begin{tabular}{|c|c|}
\hline Targeting mechanism & Reference \\
\hline Targeting ligand & {$[12][14][15]$} \\
\hline Magnetic field & {$[10][16][17]$} \\
\hline Stimuli pH responsive* & {$[11][18][19][20]$} \\
\hline *Includes pH-responsive, redox-responsive and temperature responsive.
\end{tabular}

Table 1: Examples of combined functional polymeric nanomedicine systems.

Sustained drug release at different $\mathrm{pH}$ was demonstrated with intense stable photoluminescence of the quantum dots. This nanomedicine system was found effective against B16-F10 mouse melanoma in vivo. Another way of targeted delivery is to include targeting ligands on the nanomedicine platform that will bind to the receptors on the cells for which the drug will be targeted to. It was reported that polymeric nanoaggregate functionalized with biotin for receptor-mediated drug delivery was developed combining with Chlorambucil for therapeutic action and pyrene for fluorescent imaging [12]. Some other recent advancements on combined functional polymeric nanomedicine systems were summarized in Table 1 based on different delivery mechanisms with several examples for each type. More detailed discussions about the targeted delivery mechanism can be found in the recent review [12-20].

In conclusion, recent advances of polymeric materials in nanomedicine were reviewed. The use of polymeric nanoparticles for targeted delivery, imaging and therapy was discussed. The advantages of using polymers in nanomedicine include biocompatibility, structural versatility, and low cost. With the ease to provide concurrent therapy and imaging under controlled delivery, polymeric nanoparticles have great potentials in biomedical research and applications.

\section{Acknowledgement}

The authors thank the Board of Regents of the State of Louisiana (LEQSF (2017-20)-RD-A-23) for the financial support.

\section{References}

1. Freitas RA (2005) What is nanomedicine? Nanomed Nanotech Biol Med 1: 2-9

2. Wicki A, Witzigmann D, Balasubramanian V, Huwyler J (2015) Nanomedicine in cancer therapy: Challenges, opportunities, and clinical applications. J Control Release 200: 138-157.

3. Tong R, Tang L, Ma L, Tu C, Baumgartner R, et al. (2014) Smart chemistry in polymeric nanomedicine. Chem Soc Rev 43: 6982-7012.

4. Tao L, Hu W, Liu Y, Huang G, Sumer BD, et al. (2011) Shape-specific polymeric nanomedicine: emerging opportunities and challenges. Exp Biol Med 236: 20-29.

5. Harisinghani MG, Barentsz J, Hahn PF, Deserno WM, Tabatabaei S, et al. (2003) Noninvasive Detection of Clinically Occult Lymph-Node Metastases in Prostate Cancer. N Engl J Med 348: 2491-2499. 
Citation: Wang Y (2017) Smart Polymeric Nanoparticles: Combining Targeted Delivery, Imaging and Therapy into One Nanomedicine Platform. J Bioanal Biomed 9: 269-271. doi:10.4172/1948-593X.1000191

6. Fouz MF, Mukumoto K, Averick S, Molinar O, McCartney BM, et al. (2015) Bright Fluorescent Nanotags from Bottlebrush Polymers with DNA-Tipped Bristles. ACS Cent Sci 1: 431-438.

7. Lam SJ, O'Brien-Simpson NM, Pantarat N, Sulistio A, Wong EHH, et al. (2016) Combating multidrug-resistant Gram-negative bacteria with structurally nanoengineered antimicrobial peptide polymers. Nat Microbiol 1: 16162.

8. Luk BT, Zhang L (2014) Current Advances in Polymer-Based Nanotheranostics for Cancer Treatment and Diagnosis. ACS Appl Mater Interfaces 6: 2185921873.

9. Mohan P, Rapoport N (2010) Doxorubicin as a Molecular Nanotheranostic Agent: Effect of Doxorubicin Encapsulation in Micelles or Nanoemulsions on the Ultrasound-Mediated Intracellular Delivery and Nuclear Trafficking. Mol Pharm 7: 1959-1973.

10. Roy E, Patra S, Madhuri R, Sharma PK (2017) Anisotropic Gold Nanoparticle Decorated Magnetopolymersome: An Advanced Nanocarrier for Targeted Photothermal Therapy and Dual-Mode Responsive T 1 MRI Imaging. ACS Biomater Sci Eng 3: 2120-2135.

11. Wu W, Aiello M, Zhou T, Berliner A, Banerjee P, et al. (2010) In-situ immobilization of quantum dots in polysaccharide-based nanogels for integration of optical $\mathrm{pH}$ sensing, tumor cell imaging, and drug delivery. Biomaterials 31: 3023-3031.

12. Mukherjee S, Das Sarma J, Shunmugam R (2016) pH-Sensitive Nanoaggregates for Site-Specific Drug-Delivery as Well as Cancer Cell Imaging. ACS Omega 1: 755-764.

13. Ulbrich K, Holá K, Šubr V, Bakandritsos A, Tuček J, et al. (2016) Targeted
Drug Delivery with Polymers and Magnetic Nanoparticles: Covalent and Noncovalent Approaches, Release Control, and Clinical Studies. Chem Rev 116: $5338-5431$

14. Mukherjee S, Dinda H, Chakraborty I, Bhattacharyya R, Das Sarma J, et al. (2017) Engineering Camptothecin-Derived Norbornene Polymers for Theranostic Application. ACS Omega 2: 2848-2857.

15. Kanduluru AK, Low PS (2017) Development of a Ligand-Targeted Therapeutic Agent for Neurokinin-1 Receptor Expressing Cancers. Mol Pharm.

16. Shen X, Li T, Chen Z, Geng Y, Xie X, et al. (2017) Luminescent/magnetic PLGA-based hybrid nanocomposites: a smart nanocarrier system for targeted codelivery and dual-modality imaging in cancer theranostics. Int $\mathrm{J}$ Nanomedicine 12: 4299-4322.

17. Hu X, Wang Y, Zhang L, Xu M, Zhang J, et al. (2017) Design of a pH-sensitive magnetic composite hydrogel based on salecan graft copolymer and $\mathrm{Fe}_{3} \mathrm{O}_{4} @$ $\mathrm{SiO}_{2}$ nanoparticles as drug carrier. Int. J. Biol. Macromol.

18. Shi X, Ma X, Hou M, Gao YE, Bai S, et al. (2017) pH-Responsive unimolecular micelles based on amphiphilic star-like copolymers with high drug loading for effective drug delivery and cellular imaging. J Mater Chem B 5: 6847-6859.

19. Zhao J, Yang Y, Han X, Liang C, Liu J, et al. (2017) Redox-Sensitive Nanoscale Coordination Polymers for Drug Delivery and Cancer Theranostics. ACS Appl Mater Interfaces 9: 23555-23563.

20. Zhang Z, Zhang D, Wei L, Wang X, Xu Y, et al. (2017) Temperature responsive fluorescent polymer nanoparticles (TRFNPs) for cellular imaging and controlled releasing of drug to living cells. Colloids Surf B Biointerfaces 159: 905-912. 